\title{
Nonlytic exocytosis of Cryptococcus neoformans from neutrophils in the brain vasculature
}

\author{
Xiaofan Yang ${ }^{1}$, Huijuan Wang ${ }^{2}$, Fan $\mathrm{Hu}^{3}$, Xichen Chen $^{4}$ and Mingshun Zhang ${ }^{2,5^{*}}$ (D)
}

\begin{abstract}
Background: Cryptococcus neoformans (C. neoformans) is an encapsulated budding yeast that causes life-threatening meningoencephalitis in immunocompromised individuals, especially those with acquired immunodeficiency syndrome (AIDS). To cause meningoencephalitis, C. neoformans circulating in the bloodstream must first be arrested in the brain microvasculature. Neutrophils, the most abundant phagocytes in the bloodstream and the first leukocytes to be recruited to an infection site, can ingest $C$. neoformans. Little is known about how neutrophils interact with arrested fungal cells in the brain microvasculature.
\end{abstract}

Methods: A blood-brain barrier (BBB) in vitro model was established. The interactions between neutrophils adhering to brain endothelial cells and fungi were observed under a live cell imaging microscope. A flow cytometry assay was developed to explore the mechanisms. Immunofluorescence staining of brain tissues was utilized to validate the in vitro phenomena.

Results: Using real-time imaging, we observed that neutrophils adhered to a monolayer of mouse brain endothelial cells could expel ingested C. neoformans without lysis of the neutrophils or fungi in vitro, demonstrating nonlytic exocytosis of fungal cells from neutrophils. Furthermore, nonlytic exocytosis of $C$. neoformans from neutrophils was influenced by either the fungus (capsule and viability) or the neutrophil (phagosomal pH and actin polymerization). Moreover, nonlytic exocytosis of $C$. neoformans from neutrophils was recorded in brain tissue.

Conclusion: These results highlight a novel function by which neutrophils extrude $C$. neoformans in the brain vasculature.

Keywords: C. neoformans, Neutrophils, Brain, Nonlytic exocytosis, real-time imaging

\section{Background}

Cryptococcosis is an acquired immunodeficiency virus (AIDS)-defining opportunistic infection that also occurs in organ transplant recipients and cancer patients [1]. Globally, it is estimated that there are an estimated 223, 100 cases of cryptococcosis and approximately 181,100 deaths from human immunodeficiency virus (HIV)-associated cryptococcal disease [2]. Additionally, cryptococcosis is also an infrequent fungal infection in patients with systemic lupus erythematosus [3] or tuberculosis $[4,5]$. The causative microorganism is the encapsulated fungus Cryptococcus neoformans. Fungal cells are

\footnotetext{
* Correspondence: mingshunzhang@njmu.edu.cn

${ }^{2}$ Department of Immunology, Nanjing Medical University, Nanjing 211166, China ${ }^{5} \mathrm{NHC}$ Key Laboratory of Antibody Technique, Nanjing Medical University, 101 Longmian Road, Nanjing 211166, China

Full list of author information is available at the end of the article
}

believed to enter the body through the respiratory tract and initially cause pneumonia. The most devastating event occurs when fungi disseminate into the brain via the bloodstream, consequently resulting in fatal meningoencephalitis.

Fungemia is often detected in AIDS patients during cryptococcosis [6]. Although extrapulmonary dissemination appears to be macrophage-associated [7], free fungal cells have been detected in the bloodstream [8]. These circulating fungal cells could be directly derived from the lung as free fungus or released from macrophages, as recent evidence has shown that macrophages can expel ingested C. neoformans via nonlytic exocytosis [9-11].

Recently, brain invasion by $C$. neoformans has been visualized in a mouse model based on intravital microscopy, and an important series of events that occur prior 
to transmigration into the brain has been postulated [12, 13]. The critical steps include fungal arrest in the vasculature of the brain and interaction and signaling of the fungal and endothelial cells leading to transmigration $[12,13]$. As a result of these processes, the arrested fungal cells remain within the brain vasculature for hours, providing the opportunity for immune cells circulating in the bloodstream to recognize the fungal cells. However, the intravascular interactions of immune cells with the arrested fungal cells are largely unknown. This knowledge gap limits our advances in the prevention and treatment of the illness.

Neutrophils, one of the major players during infection, are typically the first immune cells to be recruited to an infection site and are capable of eliminating microorganisms by multiple means [14]. There is evidence that neutrophils play roles in protecting the host against $C$. neoformans [15]. In vitro, neutrophils internalize C. neoformans following opsonization with complement and antibody [16-18]. In vivo, neutrophils ingest C. neoformans in the lungs of mice following intratracheal infection [19] and kill the fungi with the aid of complement C5a in the brain vasculature [20]. Previously, we reported that neutrophils may ingest and remove C. neoformans from brain vessels [21]. In this study, we recorded neutrophils expelling C. neoformans without causing lysis of either the neutrophils or fungi, which may be regulated by fungal virulence and neutrophil actin polymerization.

\section{Materials and methods}

\section{Animals}

C57BL/6 J mice from the Laboratory Animal Center, Yangzhou University (Yangzhou, China) were housed under a $12 \mathrm{~h}$ light/dark cycle in specific pathogen-free conditions with free access to mouse chow and water. Animal experiments were approved by the Laboratory Animal Ethics Committee of Nanjing Medical University (1708004).

\section{C. neoformans}

C. neoformans strain H99 was obtained from ATCC (catalog number 208821). C. neoformans B3501, Cap67, and Cap59 strains were gifts from Dr. Min Chen from the Second Military Medical University. The organisms were cultured in Sabouraud's dextrose broth (Difco) at $32{ }^{\circ} \mathrm{C}$ with gentle rotation for $16 \mathrm{~h}$ and then washed 3 times in sterile PBS (pH 7.4) before use.

\section{Nonlytic exocytosis in vitro observed under a confocal microscope}

Neutrophils were purified from bone marrow cells using a Percoll density gradient [22]. To determine the purity of neutrophils isolated from the bone marrow, the isolated cells were stained with PE anti-mouse F4/80 mAb (clone BM8, eBioscience) and Alexa Fluor 647 anti- mouse Gr-1 mAb (clone RB6-8C5, Invitrogen). As a control, cells were incubated with the corresponding isotype control antibodies. Antibodies and their ligands are listed in Additional file 1: Table S1.

To observe the dynamic interactions of neutrophils with the ingested $C$. neoformans, neutrophils purified from bone marrow cells were incubated with Alexa Fluor 647 anti-Gr-1 mAb (RB6-8C5, Invitrogen) and PE anti-mouse F4/80 mAb (BM8, eBioscience) for $30 \mathrm{~min}$. The prestained neutrophils $\left(5 \times 10^{6}\right)$ and C. neoformans labeled with FITC $\left(5 \times 10^{7}\right)$ were added to the surface of a monolayer of mouse brain endothelial cells bEnd.3 (CRL-2299, ATCC) in a glass-bottom dish (P35G-1.5-10, MatTek), which represented an in vitro BBB model [23]. E1 mAb specific for cryptococcal polysaccharide (a gift from Dr. F. Dromer, Paris, France) was added to the culture, and the cells were incubated for $1 \mathrm{~h}$ in the live cell culture equipment of the confocal microscope (LSM510, Zeiss). The dish was then extensively washed 3 times with complete medium to remove most of the nonadherent neutrophils and $C$. neoformans. The interactions of neutrophils with the ingested $C$. neoformans were imaged, and time-lapse videos were taken using a confocal microscope.

\section{Flow cytometry analysis of nonlytic exocytosis}

Flow cytometry analysis of nonlytic exocytosis was first developed by André Moraes Nicola and colleagues [11]. Briefly, neutrophils were purified from bone marrow [22] with a neutrophil isolation kit (130-097-658, Millipore) and stained with DDAO-SE $(0.5 \mu \mathrm{M}$, Invitrogen) in PBS for $10 \mathrm{~min}$ and washed 3 times. C. neoformans were stained with FITC $(1 \mathrm{mg} / \mathrm{ml}$, Sigma-Aldrich $)$ in PBS for $15 \mathrm{~min}$ and washed 3 times. Furthermore, neutrophils were stained with 7-AAD $(1 \mu \mathrm{g} / \mathrm{ml}$, Invitrogen) for $5 \mathrm{~min}$ to detect cell death, and C. neoformans were stained with Uvitex 2B (0.01\%, Polysciences Inc.) in PBS for $1 \mathrm{~min}$ to detect fungi outside of neutrophils (Additional file 2: Figure S1). DDAO-SE-stained neutrophils and FITC-stained H99 (neutrophils:H99 = 1:1) cells were incubated in the presence of the C. neoformansspecific anti-E1 antibody $(1 \mu \mathrm{g} / \mathrm{ml})$ for $2 \mathrm{~h}$ and further stained with Uvitex-2B and 7-AAD. Subsequently, FITC $^{+}$DDAO-SE ${ }^{+}$7-AAD ${ }^{-}$Unvitex $2 \mathrm{~B}^{-}$cells, which represented live neutrophils containing $C$. neoformans, were flow sorted with a BD FACS ARIA II SORP. Immediately after sorting, the cells were analyzed for purity, and $1 \times 10^{6}$ sorted neutrophils in $1 \mathrm{ml}$ of complete medium (10\% fetal calf serum in RPMI 1640 medium) were incubated in the well of a 24-well plate with or without chemicals for $2 \mathrm{~h}$. After incubation, the cells were harvested and stained with 7-AAD again for flow cytometry analysis. FITC ${ }^{-}$DDAO-SE ${ }^{+} 7-\mathrm{AAD}^{-}$cells were considered live neutrophils that had expelled the ingested $C$. neoformans. As described by Moraes Nicola and colleagues 
[11], the rate of nonlytic exocytosis was calculated based on the postsorted purity and postcultured live neutrophils not associated with C. neoformans.

\section{Immunofluorescence staining of brain tissues}

Mice were euthanized and perfused $3 \mathrm{~h}$ after i.v. infection by tail vein with $20 \times 10^{6}$ H99 cells, and the brain was removed for immunofluorescence staining. To avoid contamination of tissues by circulating fungi, perfusion was performed by injecting sterile saline $(50 \mathrm{ml})$ into the left ventricle, and the right atrium was cut open to allow drainage during the procedure [24]. The brain tissues of infected mice were prepared for frozen sections, as described previously [13]. In brief, the tissues were removed and frozen in OCT compound. Frozen tissue blocks were cut on a cryostat microtome, and 5- $\mu \mathrm{m}$ sections were placed on coated glass slides. Tissue sections were fixed in $2 \%$ neutral buffered paraformaldehyde (PFA) for $10 \mathrm{~min}$. Sections were then incubated with $2 \%$ goat serum in PBS, followed by incubation with rabbit-anti-mouse collagen IV (PA1-26148, Invitrogen), mouse-anti-cryptococcal polysaccharide (E1, a gift from Dr. F. Dromer, Paris, France), and rat-anti-mouse Ly6G (clone 1A8, Biolegend) at $4{ }^{\circ} \mathrm{C}$ overnight. After 3 washes, sections were incubated for $30 \mathrm{~min}$ with Alex Fluor 647 goat-anti-rabbit IgG $(\mathrm{H}+\mathrm{L})(\mathrm{A}-21244$, Invitrogen) to delineate brain microvasculature, Alexa Fluor 488 goatanti-mouse IgG $(\mathrm{H}+\mathrm{L})(\mathrm{A}-11001$, Invitrogen) to identify C. neoformans, and Alexa Flour 555 goat-anti-rat IgG $(\mathrm{H}+\mathrm{L})(\mathrm{A}-21434$, Invitrogen) to identify neutrophils in the brain. The sections were rinsed and mounted with glycerol and checked under a confocal microscope.

\section{Statistical analysis}

Data are expressed as the mean \pm SEM. An ANOVA was performed to establish equal variance, and a 2-tailed Student's $\mathrm{t}$ test with Bonferroni correction was applied to determine statistical significance, which was defined as $p<0.05$.

\section{Results}

Nonlytic exocytosis of $C$. neoformans from neutrophils in vitro

To address how neutrophils interact with the ingested $C$. neoformans, we first established an in vitro BBB model using the mouse brain microvasculature endothelial cell line bEnd.3. Neutrophils containing C. neoformans were seeded and attached to a monolayer of endothelial cells. Initially, we observed neutrophils containing fungi in the phagosome (Fig. 1a). At $46 \mathrm{~min}$ of image acquisition, one neutrophil started to expel the ingested fungal cell. The small yeast cell was first expelled, and the large yeast cell was also expelled in $1 \mathrm{~min}$. To confirm the exocytosis of $C$. neoformans by neutrophils without lysis, we extended our observation (Fig. 1a, Additional file 4: Movie S1) and reconstructed a z-stack of images at the end of the acquisition period. As shown in the 3D reconstruction image (Fig. 1b) and movie (Additional file 5: Movie S2), the neutrophil that contained the two fungal cells did not harbor any fungi; the two fungal cells were located close to but outside of the neutrophil. More importantly, both the neutrophils and fungi were intact. Taken together, our results demonstrate that neutrophils expel intracellular C. neoformans without lysis of both the cell itself and the fungus. Interestingly, two neighboring neutrophils containing $C$. neoformans shown in Fig. 1a, b and Additional file 4: Movie S1 did not undergo nonlytic exocytosis. Indeed, in our repeated observations $(n>20)$, nonlytic exocytosis was recorded in approximately $5 \%$ of neutrophils containing C. neoformans. Notably, less than $1 \%$ of purified cells were F4/80 positive based on the flow cytometry analysis (Fig. 1c).

Fungal capsule and viability influence nonlytic exocytosis Microscopy observations are time-consuming and ineffective in the quantification analysis. André Moraes Nicola and colleagues developed a flow cytometric method to explore the mechanisms of nonlytic exocytosis of C. neoformans from macrophages [11]. Accordingly, we sorted live neutrophils containing $C$. neoformans $\quad\left(\mathrm{FITC}^{+} \mathrm{DDAO}-\mathrm{SE}^{+}\right.$7-AAD ${ }^{-} \mathrm{Unvitex}^{-} \mathrm{B}^{-}$, Fig. $2 \mathrm{a}$ ) and calculated the frequency of nonlytic exocytosis, in which neutrophils expelled phagocytosed $C$. neoformans and became FITC negative (Fig. 2b-c). The capsule plays a vital role in the pathogenesis of $C$. neoformans infection, as acapsular strains are usually hypovirulent. In neutrophils incubated with the acapsular strain Cap67, the occurrence of nonlytic exocytosis was significantly decreased compared with that in neutrophils incubated with the wild-type B3501 strain. Similarly, nonlytic exocytosis of the acapsular strain Cap59, was also significantly reduced compared with that of the wild-type strain H99, suggesting that the capsule may regulate the nonlytic exocytosis of $C$. neoformans from neutrophils. Neutrophils killed acapsular strains more effectively than capsulated strains. Therefore, we hypothesized that fungal viability may affect nonlytic exocytosis from neutrophils. As previously reported, nonlytic exocytosis of heat-killed $C$. neoformans from macrophages is significantly suppressed $[9,10]$, and we observed that the expulsion of heat-killed C. neoformans from neutrophils was markedly decreased (Fig. 2d). In summary, nonlytic exocytosis of $C$. neoformans from neutrophils is regulated by the fungal capsule and viability.

\section{Roles of phagosome and actin in nonlytic exocytosis}

After phagocytosis, phagosomes containing the pathogens usually fuse with lysosomes and form lysophagosomes, in 

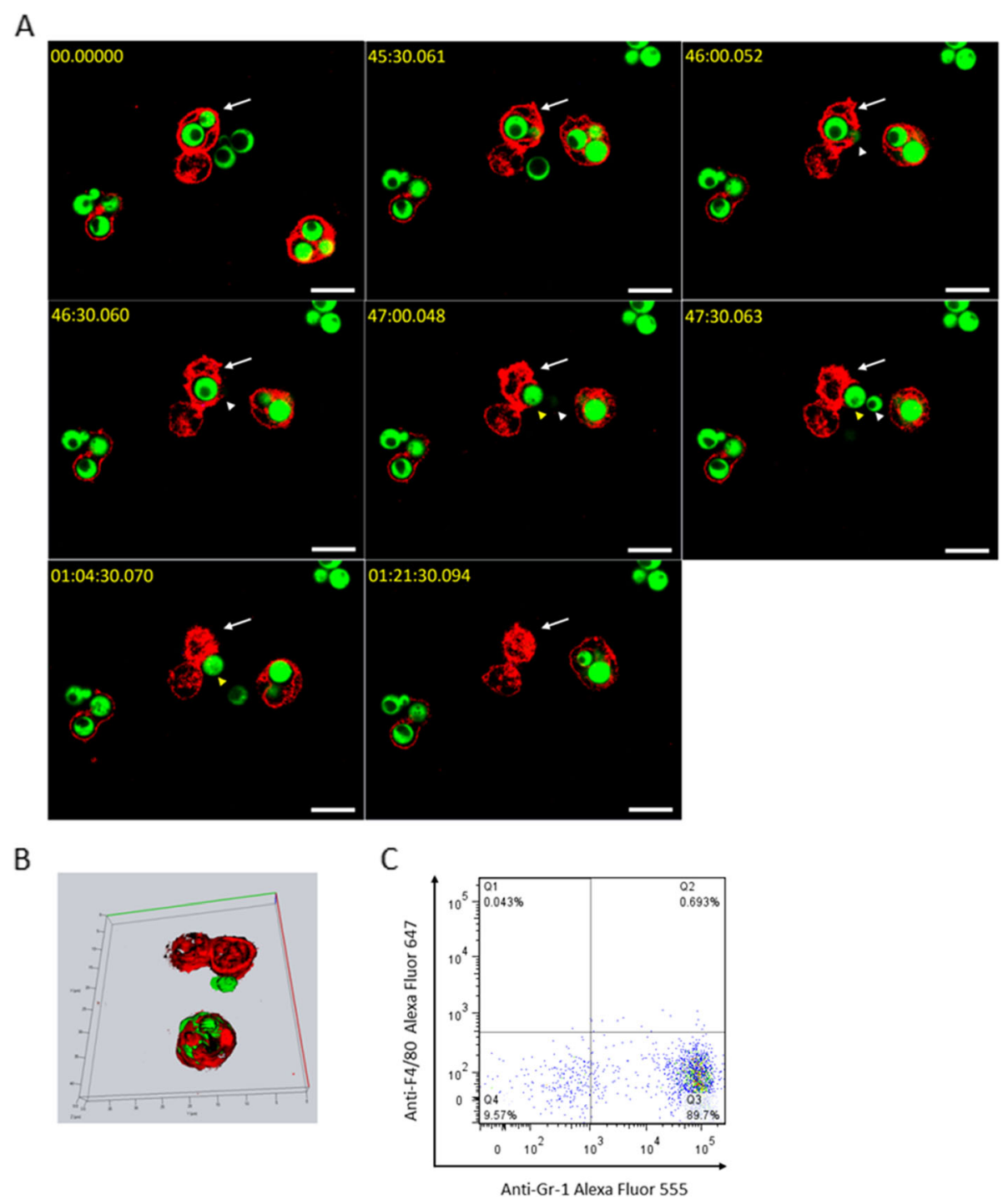

Fig. 1 Nonlytic exocytosis of $C$. neoformans by neutrophils demonstrated by time-series images and a 3D reconstruction of z-stack images. a Time-series images. C. neoformans H99 cells labeled with FITC were incubated with neutrophils, which were prestained with anti-Gr-1 (pseudocolored red) and anti-F/80 (pseudocolored blue), in a glass-bottomed dish with a precoated monolayer of mouse brain endothelial bEnd.3 cells in the presence of anti-capsule antibody E1. After $1 \mathrm{~h}$ of incubation, nonadherent cells were removed. Multichannel time-lapse fluorescence images were taken to record the dynamic process of nonlytic exocytosis of C. neoformans by the neutrophil (marked by an arrow), capturing one frame every $30 \mathrm{~s}$ for $3 \mathrm{~h}$ or until the occurrence of expulsion. At the start of image acquisition, the neutrophil contained two fungal cells. Forty-six minutes later, the phagosome fused with the cell membrane. The two fungal cells (marked by arrowheads) escaped from the neutrophil within $1 \mathrm{~min}$. b A 3D image reconstruction. To verify the expulsion of C. neoformans, z-stack images were taken at the end of the image acquisition period ( 81 min after the microscopy observation began) for the reconstruction of a 3D image of neutrophils and fungi. The neutrophils did not contain fungi; the two expelled fungal cells were located beside the "empty" neutrophil. Bar, 5 um. c Flow cytometry analysis of neutrophils. Neutrophils purified from bone marrow were stained with Alexa Flour 647 antiF4/80 and Alexa Flour 555 anti-Gr-1 or isotype control antibodies. Based on the flow cytometry analysis, approximately $90 \%$ of cells were $\mathrm{F} 4 / 80^{-} \mathrm{Gr}-1^{+}$ (neutrophils), and less than $1 \%$ of cells were $\mathrm{F} 4 / 80^{+} \mathrm{Gr}-1^{-}$(resident monocytes) or $\mathrm{F} 4 / 80^{+} \mathrm{Gr}-1^{+}$(inflammatory monocytes), indicating that the vast majority of Gr-1-positive cells in the cell preparation were neutrophils. Representative results from 3 repeated experiments

which pathogens are destroyed by various enzymes. As observed in Fig. 1, phagosomes containing fungi fused with the cell membrane before nonlytic exocytosis. The vacuolar ATPase inhibitor bafilomycin A1, which pumps protons into the phagosome and accelerates its acidification, barely changed the frequency of nonlytic exocytosis.
Treatment with the weak base chloroquine, which helps phagosome neutralization, significantly decreased the rate of nonlytic exocytosis. Since ammonium chloride, another weak base, plays an insignificant role in nonlytic exocytosis, we hypothesized that chloroquine may regulate nonlytic exocytosis via a phagosome-independent pathway. 


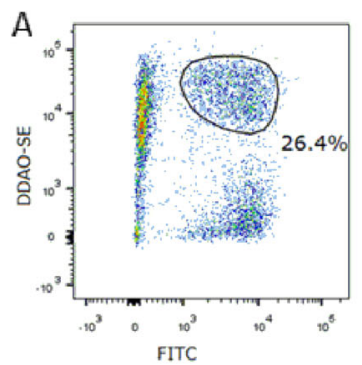

B

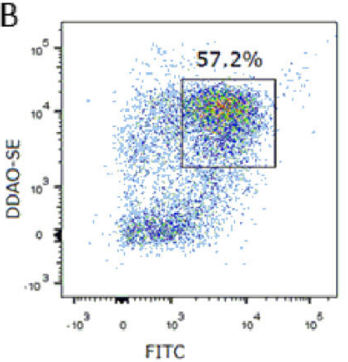

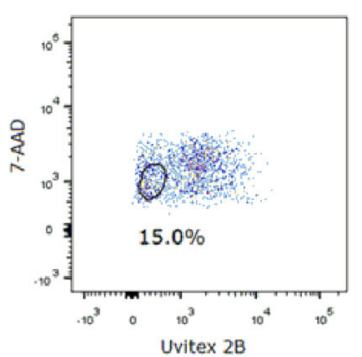

C

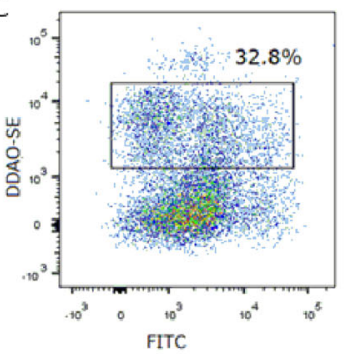

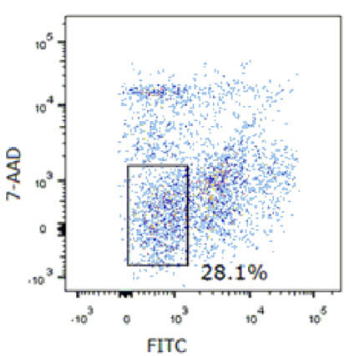

D

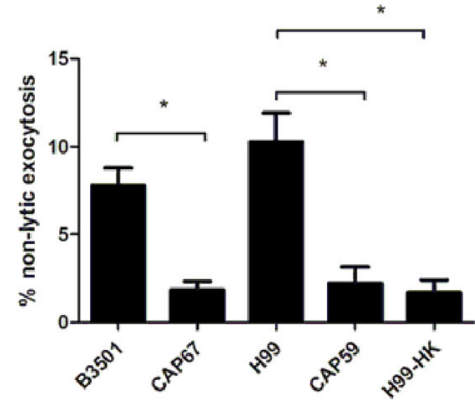

Fig. 2 Flow cytometry analysis of nonlytic exocytosis. a After incubation, live neutrophils with intracellular C. neoformans (FITC ${ }^{+}$DDAO-SE $^{+} 7$ $A A D^{-}$Unvitex $2 B^{-}$) were sorted. $\mathbf{b}$ Immediately after sorting, the cells were analyzed for purity. $\mathbf{c}$ After $2 \mathrm{~h}$ of incubation with or without drugs, neutrophils with C. neoformans were collected, stained with 7-AAD and further analyzed. FITC-DDAO-SE ${ }^{-}$7-AAD ${ }^{-}$cells were considered neutrophils undergoing nonlytic exocytosis. Representative results of 5 repeated experiments. $\mathbf{d}$ Fungal capsule and viability regulate nonlytic exocytosis. Sorted live neutrophils containing either wild-type C. neoformans (B3501, H99) or acapsular strains (Cap67, Cap59) were incubated for 2 h. Nonlytic exocytosis was calculated as described by André Moraes Nicola, mBio, 2011. Heat-killed H99 cells were included to explore whether viability affects nonlytic exocytosis. *, $p<0.05$

For example, chloroquine may exert fungicidal effects [25, 26], as evidenced by the significant increase in cytotoxicity of neutrophils against $C$. neoformans with the aid of chloroquine (Additional file 3: Figure S2). Thereafter, the dead $C$. neoformans killed by chloroquine might not be expelled from neutrophils (Fig. 3).

As observed in Fig. 1, neutrophils expelled C. neoformans cells in $2 \mathrm{~min}$. We therefore hypothesized that neutrophil actin polymerization may contribute to the nonlytic exocytosis of $C$. neoformans. In the expulsion of C. neoformans from macrophages, the actin flash was inversely correlated with nonlytic exocytosis [27]. Therefore, we tested whether chemicals interfering with actin flashes play roles in nonlytic neutrophil exocytosis. As shown in Fig. 3, treatment with the actin polymerization inhibitor cytochalasin D significantly enhanced the nonlytic exocytosis of $C$. neoformans from neutrophils.
Moreover, treatment with the Arp2/3 complex-mediated actin polymerization inhibitor wiskostatin also increased C. neoformans expulsion from neutrophils, although to a lesser extent. In contrast, treatment with jasplakinolide, a potent actin polymerization inducer, had a reduced effect on the nonlytic exocytosis. Collectively, these results show that classical WASP-Arp2/3 complex-mediated actin filament nucleation may play a role in the nonlytic exocytosis of $C$. neoformans from neutrophils.

\section{Nonlytic exocytosis of $C$. neoformans from neutrophils in the brain vasculature}

To determine whether neutrophils recognize $C$. neoformans in the brain in vivo, we first recorded the intravascular interactions of neutrophils with $C$. neoformans in the brains of living mice using intravital microscopy. Gr1 is predominantly expressed on mouse neutrophils and 


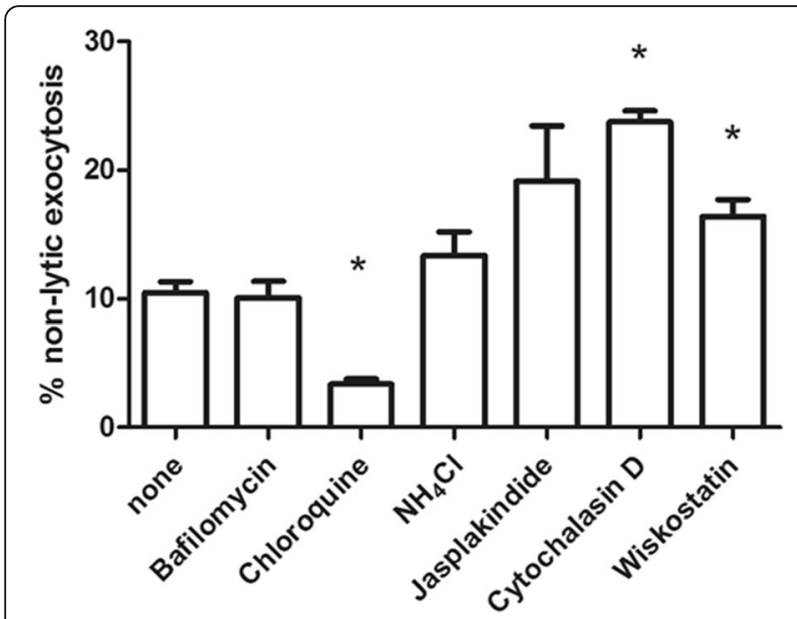

Fig. 3 Phagosomes and actin polymerization regulate nonlytic exocytosis. Live neutrophils containing H99 cells were treated with various chemicals for $2 \mathrm{~h}$, and the rate of nonlytic exocytosis was calculated. Bafilomycin A1 (100 nM), vacuolar ATPase inhibitor; chloroquine $(10 \mu \mathrm{M})$ and ammonium chloride $(20 \mathrm{mM})$, weak bases; jasplakinolide $(50 \mathrm{nM})$, actin depolymerization inhibitor; cytochalasin $(10 \mathrm{nM})$, actin polymerization inhibitor; and wiskostatin $(100 \mathrm{nM})$, Arp2/3 complex-mediated actin polymerization inhibitor. *,$p<0.05$ is also expressed on inflammatory monocytes [28]. However, the vast majority of $\mathrm{Gr}-1^{+}$cells in the circulation are neutrophils, which are the most abundant white blood cell type in circulation. Therefore, anti-Gr-1 mAbs have been extensively used to label mouse neutrophils in intravital imaging [29]. Nevertheless, in this study, we also injected the mice with anti-F4/80 mAb to exclude the tiny population of $\mathrm{Gr}-1^{+}$monocytes from neutrophils, as $\mathrm{Gr}-1^{+}$ monocytes also express F4/80. To determine whether neutrophils expel $C$. neoformans in the brain in vivo, we recorded the intravascular interactions of neutrophils with C. neoformans in the brains using immunohistochemistry. As shown in Fig. 4a, intravascular neutrophils engulfed $C$. neoformans in the brain. As expected, a neutrophil attached to the brain capillary expelled $C$. neoformans (Fig. 4b-c, Additional file 6: Movie S3). Therefore, we provide direct evidence that nonlytic exocytosis of $C$. neoformans from neutrophils occurs in the brain vasculature.

\section{Discussion}

As the most commonly encountered clinical manifestation of cryptococcosis, cryptococcal meningoencephalitis is a

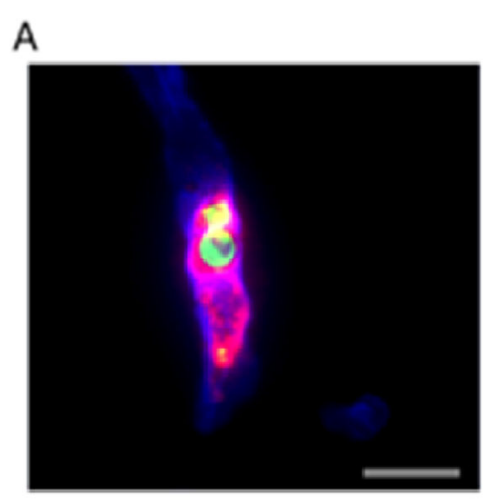

B

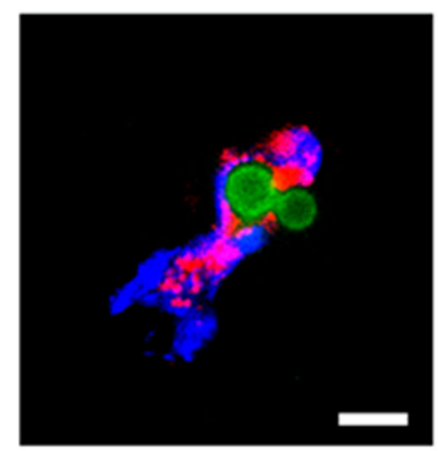

C

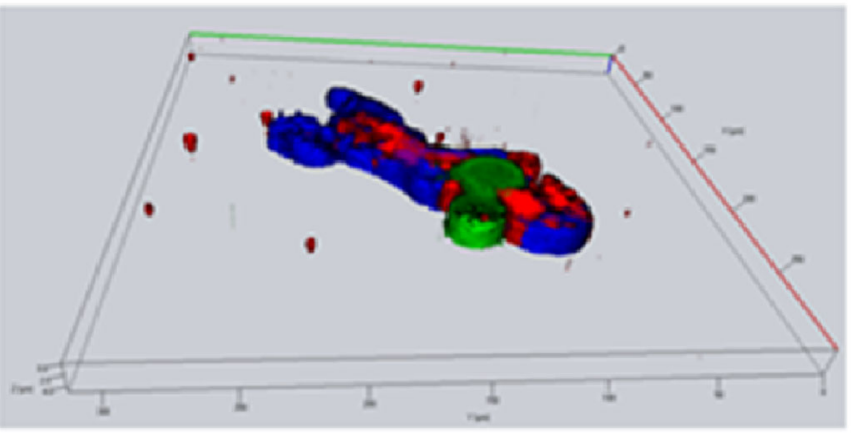

Fig. 4 Neutrophils expel C. neoformans in the brain vasculature. Neutrophils were stained with anti-Ly6G (pseudocolored red); C. neoformans was stained with the anti-capsule antibody E1 (pseudocolored green), and the brain vasculature was stained with anti-collagen IV (pseudocolored blue). a Neutrophils containing C. neoformans in the brain vasculature; original scale bar, $20 \mu \mathrm{m} ; \mathbf{b}$ Two fungal cells located in a neutrophil attached to a brain capillary. One fungal cell seemed to be expelled from the neutrophil; original scale bar, $10 \mu \mathrm{m}$. c A 3D reconstructed image based on z-stack images was captured for further analysis 
devastating infectious disease. In AIDS patients, the mortality rate for cryptococcal meningoencephalitis was approximately $20 \%$ [30]. Once arrested in the brain vasculature, $C$. neoformans may transmigrate across the $\mathrm{BBB}$ and proliferate in the brain parenchyma. Therefore, it is imperative to explore how immune cells respond to $C$. neoformans in the brain [31]. Depletion of phagocytes, including macrophages and neutrophils, may impair the infiltration of $C$. neoformans into the brain [32]. It has been established that macrophages [9-11] and monocytes [33] can expel intracellular C. neoformans. In the present study, we documented that nonlytic exocytosis of $C$. neoformans from neutrophils occurs in vitro and in vivo.

Neutrophils and macrophages originate from common myeloid progenitors. Neutrophils, however, are different from macrophages. First, neutrophils are considered key responders to infection. Once released into the bloodstream, $C$. neoformans transmigrate across the BBB within $6 \mathrm{~h}$ [24]. Therefore, an immediate immune response is indispensable for the clearance or dissemination of $C$. neoformans brain infections. Indeed, intravascular clearance of C. neoformans is largely dependent on neutrophils [20]. Second, the half-life of neutrophils is much shorter than that of macrophages. In ex vivo culture, neutrophils may die within $24 \mathrm{~h}$. The average lifespan for circulating neutrophils, however, is as long as 5.4 days in vivo [34], raising the possibility that neutrophils may not be as fragile as expected. In the present study, neutrophils cocultured with endothelial cells and $C$. neoformans were observed and analyzed within several hours. As microbes may regulate the aging of neutrophils [35], we hypothesize that C. neoformans may affect the lifespan of neutrophils. Third, the neutrophil phagosome may be acidification independent [36]. In the nonlytic exocytosis of $C$. neoformans from macrophages [37], phagosomal $\mathrm{pH}$ modulation was found to be associated with exocytosis. In neutrophils, phagosomal $\mathrm{pH}$ modification, either with vacuolar ATPase inhibitor bafilomycin A1 or ammonium chloride treatment, had little effect on the nonlytic exocytosis of C. neoformans. The effect of chloroquine on the occurrence of nonlytic exocytosis was largely related to the regulation of fungal viability rather than phagosomal acidification. Similar observations were observed for the capsule-deficient strains or heat-killed fungi.

Previously, we demonstrated that neutrophils may phagocytose arrested $C$. neoformans attached to the brain vasculature, which may help alleviate brain infections [21]. In this study, we provide evidence that neutrophils expel intracellular $C$. neoformans in vitro and in the brain vasculature. We hypothesize that the role of neutrophils may be a double-edged sword, i.e., both protective and deleterious, during the course of cryptococcosis. On the one hand, neutrophils are important for killing [16] or removing C. neoformans arrested in the brain microvasculature; on the other hand, neutrophils carry $C$. neoformans and exocytose them in the brain vasculature, contributing to the brain infection.

Our study has some notable limitations. The nonlytic exocytosis of $C$. neoformans from monocytes and macrophages may originate from environmental interactions [38, 39]. In contrast, the evolutionary role of nonlytic exocytosis of C. neoformans from neutrophil is unknown. Moreover, neutrophils are heterogeneous with many different functions [40]. Neutrophils with nonlytic exocytosis capabilities and the mechanisms warrant improved definitions.

\section{Conclusion}

Our results suggest that neutrophils adhering to brain vasculature extrude $C$. neoformans via nonlytic exocytosis, which is regulated by fungal virulence factors (viability and capsule) and neutrophils (phagosome and actin). The mechanisms behind the nonlytic exocytosis of $C$. neoformans from neutrophils in the brain vasculature require further study.

\section{Additional files}

Additional file 1: Table S1. Antibodies in the study. (DOCX 14 kb)

Additional file 2: Figure S1. Flow cytometry analysis of neutrophils and C. neoformans stained with various fluorophores. Bone marrow-derived neutrophils were stained with DDAO-SE (0.5 $\mathrm{MM}$, Invitrogen) in PBS for 10 min and washed 3 times. C. neoformans cells were stained with FITC (1 mg/ml, Sigma-Aldrich) in PBS for $15 \mathrm{~min}$ and washed 3 times. Furthermore, neutrophils were stained with 7-AAD (1 $\mathrm{\mu g} / \mathrm{ml}$, Invitrogen) for $5 \mathrm{~min}$ to detect cell death, and $C$. neoformans were stained with Uvitex 2B (0.01\%, Polysciences Inc.) in PBS for 1 min to detect extracellular fungi. Representative results of 5 repeated experiments. (TIF $575 \mathrm{~kb}$ )

Additional file 3: Figure S2. Chloroquine increased the fungicidal activity of neutrophils against $C$. neoformans. In a 96-well plate, $C$. neoformans $\left(5 \times 10^{3}\right.$ cells per well) were incubated alone or with neutrophils $\left(1 \times 10^{5}\right.$ cells per well) and chloroquine $(10 \mu \mathrm{M}$, working concentration) for 4 h. After incubation, the live fungi were quantified on YPA agar. ${ }^{*}, p<0.05$. (TIF 235 kb)

Additional file 4: Movie S1. FITC-labeled C. neoformans H99 were incubated with anti-Gr-1 (pseudocolored red) and anti-F4/80 (pseudocolored blue) prestained neutrophils in a glass-bottomed dish with a precoated monolayer of mouse brain endothelial bEnd.3 cells in the presence of anti-capsule antibody E1. After $1 \mathrm{~h}$ of incubation, nonadherent cells were removed. Multichannel time-lapse fluorescence images (oil-immersion objective lens) were captured to record the dynamic process of nonlytic exocytosis of $C$. neoformans by the neutrophil (marked by an arrow), with one frame captured every $30 \mathrm{~s}$ for $3 \mathrm{~h}$ or until the occurrence of expulsion. The time-series images were exported as a video at 5 frames per second. Also refer to Fig. 1. (MOV $1612 \mathrm{~kb}$ )

Additional file 5: Movie S2. A 3D reconstruction from z-stack images taken at the end of the image acquisition period (81 min after the microscopy observation began) showing the "empty" neutrophil (pseudocolored red) and the two expelled fungal cells (pseudocolored green) beside the neutrophil. Also refer to Fig. 1. (MOV 8570 kb)

Additional file 6: Movie S3. A 3D movie reconstructed from z-stack images of immunofluorescence stained cells showing the nonlytic exocytosis of $C$. neoformans from neutrophils in the brain vasculature. Also refer to Fig. 4 (MOV $2676 \mathrm{~kb}$ ) 


\section{Abbreviations}

AIDS: Acquired immunodeficiency syndrome; BBB: Blood-brain barrier; C. neoformans: Cryptococcus neoformans; HIV: Human immunodeficiency virus; PFA: Paraformaldehyde

\section{Acknowledgments}

Not Applicable.

\section{Authors' contributions}

$M Z$ designed and supervised the experiments; $X Y, X C$ and HF carried out the experiments; $X Y$ and HW carried out the statistical analyses, and edited figures and the manuscript. All the authors reviewed the paper. All authors read and approved the final manuscript.

\section{Funding}

This work was supported by a National Natural Science Foundation of China grant 81671563 (to MZ)

\section{Availability of data and materials}

All data generated or analyzed during this study are included in this published article (and its Supplementary Information files).

\section{Ethics approval and consent to participate}

All animal protocols were reviewed and approved by the Animal Care and Use Committee (IACUC) of Nanjing Medical University (1708004). The Experimental Animal Care and Use Guidelines from the Ministry of Science and Technology of China (MOST-2011-02) were strictly followed.

\section{Consent for publication}

Not Applicable.

\section{Competing interests}

The authors declare that they have no competing interests.

\section{Author details}

${ }^{1}$ The Laboratory Center for Basic Medical Sciences, Nanjing Medical University, Nanjing 211166, China. ²Department of Immunology, Nanjing Medical University, Nanjing 211166, China. ${ }^{3}$ State Key Laboratory of Reproductive Medicine, Nanjing Medical University, Nanjing 211166, China. ${ }^{4}$ Analysis center, Nanjing Medical University, Nanjing 211166, China. ${ }^{5} \mathrm{NHC}$ Key Laboratory of Antibody Technique, Nanjing Medical University, 101 Longmian Road, Nanjing 211166, China.

Received: 25 May 2019 Accepted: 29 August 2019

Published online: 09 September 2019

\section{References}

1. Kwon-Chung KJ, Sorrell TC, Dromer F, Fung E, Levitz SM. Cryptococcosis: clinical and biological aspects. Med Mycol. 2000;38(Suppl 1):205-13.

2. Rajasingham R, Smith RM, Park BJ, Jarvis JN, Govender NP, Chiller TM, et al. Global burden of disease of HIV-associated cryptococcal meningitis: an updated analysis. Lancet Infect Dis. 2017;17(8):873-81.

3. Fang W, Chen M, Liu J, Hagen F, Ms A, Al H, et al. Cryptococcal meningitis in systemic lupus erythematosus patients: pooled analysis and systematic review. Emerg Microbes Infect. 2016;5(9):e95

4. Mete B, Saltoglu N, Vanli E, Ozkara C, Arslan F, Mert A, et al. Simultaneous cryptococcal and tuberculous meningitis in a patient with systemic lupus erythematosus. J Microbiol Immunol Infect. 2016:49(2):289-94.

5. Chen M, Al-Hatmi AM, Chen Y, Ying Y, Fang W, Xu J, et al. Cryptococcosis and tuberculosis co-infection in mainland China. Emerg Microbes Infect 2016;5(9):e98.

6. Chretien F, Lortholary O, Kansau I, Neuville S, Gray F, Dromer F. Pathogenesis of cerebral Cryptococcus neoformans infection after fungemia. J Infect Dis. 2002;186(4):522-30

7. Casadevall A. Cryptococci at the brain gate: break and enter or use a Trojan horse? J Clin Investig. 2010;120(5):1389-92.

8. Santangelo R, Zoellner H, Sorrell T, Wilson C, Donald C, Djordjevic J, et al. Role of extracellular phospholipases and mononuclear phagocytes in dissemination of cryptococcosis in a murine model. Infect Immun. 2004 72(4):2229-39.
9. Alvarez M, Casadevall A. Phagosome extrusion and host-cell survival after Cryptococcus neoformans phagocytosis by macrophages. Curr Biol. 2006; 16(21):2161-5

10. Ma H, Croudace JE, Lammas DA, May RC. Expulsion of live pathogenic yeast by macrophages. Curr Biol. 2006;16(21):2156-60.

11. Nicola AM, Robertson EJ, Albuquerque P, Derengowski Lda S, Casadevall A. Nonlytic exocytosis of Cryptococcus neoformans from macrophages occurs in vivo and is influenced by phagosomal pH. MBio. 2011;2(4):e00167-11. https://www.ncbi.nlm.nih.gov/pmc/articles/PMC3150755/.

12. Shi $\mathrm{M}$, Colarusso $\mathrm{P}, \mathrm{Mody} \mathrm{CH}$. Real-time in vivo imaging of fungal migration to the central nervous system. Cell Microbiol. 2012:14(12):1819-27.

13. Shi M, Li SS, Zheng C, Jones GJ, Kim KS, Zhou H, et al. Real-time imaging of trapping and urease-dependent transmigration of Cryptococcus neoformans in mouse brain. J Clin Invest. 2010;120(5):1683-93.

14. Hickey MJ, Kubes P. Intravascular immunity: the host-pathogen encounter in blood vessels. Nat Rev Immunol. 2009;9(5):364-75.

15. Zhang M, Sun D, Shi M. Dancing cheek to cheek: Cryptococcus neoformans and phagocytes. Springerplus. 2015;4:410.

16. Kozel TR, Highison B, Stratton CJ. Localization on encapsulated Cryptococcus neoformans of serum components opsonic for phagocytosis by macrophages and neutrophils. Infect Immun. 1984;43(2):574-9.

17. Voelz K, May RC. Cryptococcal interactions with the host immune system. Eukaryot Cell. 2010;9(6):835-46.

18. Sun D, Zhang M, Liu G, Wu H, Zhu X, Zhou H, et al. Real-time imaging of interactions of neutrophils with Cryptococcus neoformans demonstrates a crucial role of complement C5a-C5aR signaling. Infect Immun. 2015;84(1):216-29.

19. Feldmesser M, Kress $Y$, Novikoff $P$, Casadevall A. Cryptococcus neoformans is a facultative intracellular pathogen in murine pulmonary infection. Infect Immun. 2000:68(7):4225-37.

20. Sun D, Zhang M, Liu G, Wu H, Li C, Zhou H, et al. Intravascular clearance of disseminating Cryptococcus neoformans in the brain can be improved by enhancing neutrophil recruitment in mice. Eur J Immunol. 2016;46(7):1704-14.

21. Zhang M, Sun D, Liu G, Wu H, Zhou H, Shi M. Real-time in vivo imaging reveals the ability of neutrophils to remove Cryptococcus neoformans directly from the brain vasculature. J Leukoc Biol. 2016;99(3):467-73.

22. Boxio R, Bossenmeyer-Pourie C, Steinckwich N, Dournon C, Nusse O. Mouse bone marrow contains large numbers of functionally competent neutrophils. J Leukoc Biol. 2004:75(4):604-11.

23. Koto T, Takubo K, Ishida S, Shinoda H, Inoue M, Tsubota K, et al. Hypoxia disrupts the barrier function of neural blood vessels through changes in the expression of claudin-5 in endothelial cells. Am J Pathol. 2007;170(4):1389-97.

24. Charlier C, Chretien F, Baudrimont M, Mordelet E, Lortholary O, Dromer F. Capsule structure changes associated with Cryptococcus neoformans crossing of the blood-brain barrier. Am J Pathol. 2005;166(2):421-32.

25. Harrison TSGG, Levitz SM. Conditional lethality of the diprotic weak bases chloroquine and quinacrine against Cryptococcus neoformans. J Infect Dis. 2000:182:283-9.

26. Levitz SM, Harrison TS, Tabuni A, Liu X. Chloroquine induces human mononuclear phagocytes to inhibit and kill Cryptococcus neoformans by a mechanism independent of iron deprivation. J Clin Invest. 1997; 100(6):1640-6.

27. Johnston SA, May RC. The human fungal pathogen Cryptococcus neoformans escapes macrophages by a phagosome emptying mechanism that is inhibited by Arp2/3 complex-mediated actin polymerisation. PLoS Pathog. 2010;6(8):e1001041.

28. Geissmann F, Jung S, Littman DR. Blood monocytes consist of two principal subsets with distinct migratory properties. Immunity. 2003; 19(1):71-82.

29. Cools-Lartigue J, Spicer J, McDonald B, Gowing S, Chow S, Giannias B, et al. Neutrophil extracellular traps sequester circulating tumor cells and promote metastasis. J Clin Invest. 2013;123(8):3446-58. https://www.ncbi.nlm.nih.gov/ pmc/articles/PMC3726160/.

30. van Spil WE, Nooijen S, de Jong PY, Aliredjo RP, de Sevaux RG, Verhave JC. Cryptococcal meningitis. Ned Tijdschr Geneeskd. 2015;159:A8478.

31. Shi M, Mody CH. Fungal infection in the brain: what we learned from Intravital imaging. Front Immunol. 2016;7:292.

32. Kaufman-Francis K, Djordjevic JT, Juillard PG, Lev S, Desmarini D, Grau GER, et al. The early innate immune response to, and phagocyte-dependent entry of, Cryptococcus neoformans map to the perivascular space of cortical post-capillary Venules in Neurocryptococcosis. Am J Pathol. 2018; 188(7):1653-65. 
33. Alvarez M, Burn T, Luo Y, Pirofski LA, Casadevall A. The outcome of Cryptococcus neoformans intracellular pathogenesis in human monocytes. BMC Microbiol. 2009;9:51.

34. Pillay J, den Braber I, Vrisekoop N, Kwast LM, de Boer RJ, Borghans JAM, et al. In vivo labeling with ( $\mathrm{H} 2 \mathrm{O})-\mathrm{H}-2$ reveals a human neutrophil lifespan of 5 . 4 days. Blood. 2010;116(4):625-7.

35. Zhang DC, Chen G, Manwani D, Mortha A, Xu CL, Faith JJ, et al. Neutrophil ageing is regulated by the microbiome. Nature. 2015;525(7570):528-32.

36. Nordenfelt P, Tapper H. Phagosome dynamics during phagocytosis by neutrophils. J Leukoc Biol. 2011;90(2):271-84.

37. Fu MS, Coelho C, De Leon-Rodriguez CM, Rossi DCP, Camacho E, Jung EH, et al. Cryptococcus neoformans urease affects the outcome of intracellular pathogenesis by modulating phagolysosomal pH. PLoS Pathog. 2018;14(6): e1007144

38. Chrisman CJ, Alvarez M, Casadevall A. Phagocytosis of Cryptococcus neoformans by, and nonlytic exocytosis from, Acanthamoeba castellanii. Appl Environ Microbiol. 2010;76(18):6056-62.

39. Watkins RA, Andrews A, Wynn C, Barisch C, King JS, Johnston SA. Cryptococcus neoformans escape from Dictyostelium Amoeba by both WASH-mediated constitutive exocytosis and Vomocytosis. Front Cell Infect Microbiol. 2018;8:108.

40. Bouma G, Ancliff PJ, Thrasher AJ, Burns SO. Recent advances in the understanding of genetic defects of neutrophil number and function. Brit J Haematol. 2010;151(4):312-26.

\section{Publisher's Note}

Springer Nature remains neutral with regard to jurisdictional claims in published maps and institutional affiliations.

Ready to submit your research? Choose BMC and benefit from:

- fast, convenient online submission

- thorough peer review by experienced researchers in your field

- rapid publication on acceptance

- support for research data, including large and complex data types

- gold Open Access which fosters wider collaboration and increased citations

- maximum visibility for your research: over $100 \mathrm{M}$ website views per year

At $\mathrm{BMC}$, research is always in progress.

Learn more biomedcentral.com/submissions 